\title{
Neuroprotective, Behavioural and Biochemical Effects of Aqueous Crude Root Extract of Salacia reticulata on Aluminium Chloride Induced Damage in the Cerebral Cortex of Adult Male Wistar Rats
}

\author{
Elvis Tams Godam ${ }^{1 *}$, Sunday S Musa ${ }^{2}$, Peter S Akpulu² and Progress D Victor ${ }^{1}$ \\ ${ }^{1}$ Department of Human Anatomy, Faculty of Basic Medical Sciences, College of Medical Sciences, Rivers State University, Nkpolu-Oroworukwo, \\ Port Harcourt, Nigeria \\ ${ }^{2}$ Department of Human Anatomy, Faculty of Basic Medical Sciences, Ahmadu Bello University Zaria, Kaduna State, Nigeria \\ *Corresponding Author: Elvis Tams Godam, Department of Human Anatomy, Faculty of Basic Medical Sciences, College of Medical \\ Sciences, Rivers State University, Port Harcourt, Rivers State Nigeria.
}

Received: January 07, 2019; Published: March 13, 2019

DOI: $10.31080 /$ ASCB.2019.03.0115

\begin{abstract}
Heavy metals like aluminium chloride has proven to have an effect with learning, understanding, memory, concentration and decision making, which affect everyday life of people in urban areas. The study was designed to ascertain if Salacia reticulate can protect the neurons of the cerebral cortex, alleviate anxiety, as well as protect the liver. Twenty-five (25) male wistar rats were randomly selected and divided into five groups of five (5) animals each as follows: Group A: Normal control; Group B: Alcl ${ }_{3}$ control; Group C: $\mathrm{Alcl}_{3}+250 \mathrm{mg} / \mathrm{kgbw}$ salacia reticulata; Group D: $\mathrm{Alcl}_{3}+300 \mathrm{mg} / \mathrm{kgbw}$ salacia reticulate; Group E: $\mathrm{Alcl}_{3}+$ Vitamin E. The following tests were carried out: Y-Maze test, Liver enzymes test and histopathological test. The results from the Y-maze test showed decrease in the meantime taken by the animals to make alternations in the aluminium chloride treated group compared to the normal control group, implying that $\mathrm{Alcl}_{3}$ induced anxiety. The meantime increased in groups treated with Salacia reticulata. Histological examination showed full neuronal damage in the $\mathrm{Alcl}_{3}$ treated group only, partial restoration of cellular and neutrophil profile was seen in the low dose groups treated with Salacia reticulata, restoration of cellular and neutrophil profile was seen in groups treated with high doses of Salacia reticulata. In Alcl ${ }_{3}$ treated group, AST, ALT, ALP increased rapidly, while in Salacia reticulata and vitamin E treated groups, the level of these enzymes where reduced. From the results obtained, Aqueous Crude Root Extract of Salacia reticulata has Neuroprotective properties, it is capable of alleviating anxiety and stabilizing liver enzymes.

Keywords: Neuroprotective; Salacia reticulate; AlCl3; Anxiety; Cerebral Cortex; Wistar Rats
\end{abstract}

\section{Introduction}

The cerebral cortex is a sheet of neural tissue that is outer-most to the cerebrum of the mammalian brain. The cerebral cortex has been reported to be the biggest part of the brain. This large and complicated neural circuit is involved in most of the brain's highest functions, such as memory, language and sight. In man and higher animals, modifications of behaviour are due to cortical activity [1]. It also integrates higher mental functions, general movement, visceral functions, and behavioural reactions. The cerebral cortex contains motor areas, which control complex motor skills and some involuntary movements; sensory areas, which receive information from the sense organs; and association areas, which are responsible for thought, learning language, and personality $[2,3]$.
Heavy metals are potentially toxic to human and can lead to neural diseases [4]. Some researchers have reported that high concentrations of heavy metals (lead, arsenic) damages the nervous, endocrine, circulatory and excretory system [4].

Some authors have reported that drinking water with heavy metal toxicity has fatal effects which include reduced mental and central nervous function. They also cause irregularity in blood composition, badly effect vital organs such as kidneys and liver [5]. The long-term exposure of these metals result in physical, muscular, neurological degenerative processes that cause Alzheimer's disease (brain disorder), Parkinson's disease (degenerative disease of the brain), muscular dystrophy (progressive skeletal muscle weakness), multiple sclerosis (a nervous system disease that affects 
brain and spinal cord), Also, Lead is one of the most common heavy metal in drinking water, if occurred more than its permissible limit shows general metabolic poison and enzyme inhibitor [6]. Heavy metals like aluminium chloride has proven to have an effect with learning, understanding, memory, concentration and decision making, which affect everyday life of people in urban areas.

Results from this study, can be used to improve cognitive impairment, neurobehavioral and memory burden in subjects that are been exposed to heavy metals in rural and urban areas in Nigeria. And Africa at large.

\section{Materials and Methods \\ Reagent and Materials}

The Reagents that were used are 10\% formal calcium, $10 \%$ formal saline, formalin, methylated spirit, cotton wool, lab coat, sterile surgical blades, table top, hand gloves, face mask water bath, light microscope, memory card, digital camera, aqueous extract, Bouin's fluid, H\&E stain, Bielschowsky stain.

\section{Equipment}

Equipment's used for this experiment are; cages, water rubbers, Ymaze, wistar rats, weighing scale, pencil, rotary microtome, slides, cover slips, Uranium (235U), light water, Beryllium, Nitric acid, gamma-ray data acquisition system, A Multi-Purpose GammaRay, lab coat, water bath, light microscope, memory card, digital camera, Bouin's fluid, H\&E stain, Bielschowsky stain.

\section{Collection and extraction of plant}

The plant (Salacia reticulate) was harvested from Agbonchia, Nchia, in Eleme local Government in Rivers state, Nigeria. The U.S Gallah of institute of biochemical Research NARICT Zaria, Kaduna state, Nigeria, verified the plant. Extraction was carried out using water.

\section{Procedure for phytochemical screening}

$50 \%$ of the crude extract was partitioned using petroleum, ether, benzene and ethyl acetate. And the remaining left was dissolved in methanol; all fractions obtained were subjected to phytochemical screening employing the standard screening test [7].

\section{Experimental protocol}

Twenty-five (25) male wistar rats were randomly selected and divided into five groups of five (5) animals each as follows:

\begin{tabular}{|c|c|c|c|}
\hline $\begin{array}{l}\text { Groups } \\
(n=5)\end{array}$ & Duration & Treatment & Administration \\
\hline A & 14days & Normal saline & Oral \\
\hline B & 14days & $\mathrm{AlCl}_{3\left(\begin{array}{c}65 \mathrm{mg} / \mathrm{kg} \mathrm{bw}) \\
\text { orally }\end{array}\right.}$ & Oral \\
\hline $\mathrm{C}$ & 14days & $\begin{array}{c}\mathrm{AlCl}_{3} 65 \mathrm{mg} / \mathrm{kg} \mathrm{bw} \\
\text { single dose })+S \\
\text { reticulata } 250 \mathrm{mg} / \mathrm{kgbw} \\
\text { orally }\end{array}$ & Oral \\
\hline $\mathrm{D}$ & 14days & $\begin{array}{c}\mathrm{AlCl}_{3 \text { ( }} 65 \mathrm{mg} / \mathrm{kg} \text { bw for } \\
7 \text { days) single dose } \\
\text { orally } \\
+ \text { S. reticulata } 300 \mathrm{mg} / \\
\text { kgbw for } 7 \text { days }\end{array}$ & Oral \\
\hline$E$ & 14days & $\begin{array}{l}\mathrm{AlCl}_{3}(65 \mathrm{mg} / \mathrm{kg} \text { bw } \\
\text { orally })+ \text { Vitamin E } \\
250 \mathrm{mg} / \mathrm{kgbw} \text { orally }\end{array}$ & Oral \\
\hline
\end{tabular}

Table 1

Learning and memory (Y maze alternation Test)

Learning and memory test was carried out in a Y-maze with three [3] white opaque plastic arms at an angle of $120^{\circ}$ from each other, labelled A, B, C respectively. This Y-maze test measures the ability of experimental animals to explore new environments; parts of the brain including the hippocampus, septum, basal forebrain and prefrontal cortex- are involved in this task [8]. Modified forced alternation test was used as protocol [8].

Learning and memory will have carried out three times for each group on the first day of administration, $7^{\text {th }}$ day of administration and final day of administration. Learning and memory test using wistar rats was carried out three days of animal handling. The test involves two steps (sample trial and retrieval trial), each of these steps requires five minutes. For the aluminium experiment, wistar rats were doused with aluminium chloride before sample trial. In step one (sample trial), the wistar rats were placed at the end of the start arm, facing the wall and away from the Centre. And later allowed to explore two arms of the Y-maze, while entry into the third arm was blocked.

At the end of the first step, rats were returned to its home cage for a $30 \mathrm{~min}$ inter-trial interval. In the second step, the block in arm 3 was removed; the rats were again placed into the start arm, and then allowed to access all three arms of the maze. Any 
rat that climbs on the maze wall was immediately returned into the abandoned maze arm. Inorder to prevent odour cues, between step one and step two, the maze was wiped with a Quatricide ${ }^{\circledR}$ dilution. When $85 \%$ of a rat's body entered one arm, it is recorded as an arm entry. While the time spent in the novel arm divided by the time spent in all arms during the retrieval trial (second step) was recorded as Novel Arm. The number of rats in percentage entering first the novel arm during second step was recorded as Forced Alternation (\%). Rats that were unable to explore three arm entries within the first sixty seconds of the second step of the test were excluded from the analysis [8].

\section{Animal sacrifice}

At the end of the experiment, body weight of the rats was obtained using a digital weighing balance; the animals were anaesthetize using chloroform and humanely sacrificed. Brain tissues were assessed by opening the sutures of the skull.

\section{Biochemical analysis}

The serum was centrifuged and analysed to quantify liver enzymes, (ALT, AST, and ALP).

Protein, albumin and globulin presence were also analysed using an autoanalyzer machine.

\section{Histological analysis}

Harvested brain tissue was fixed in $10 \%$ formal calcium. Histological staining of samples was the carried out in the Department of Human Anatomy, ABU, Zaria. These tissues were stained using Hematoxylin and Eosin for general histological architecture. The special stain used for neurological cells and fibers was Bielchowsky"s silvers stain.

The slides were mounted and studied using an Olympus microscope. And photomicrographs were taken with the aid of a digital camera (Amscope, MD 900). Digital camera was placed in the eyepiece of an Olympus microscope, image was taken using application installed in a Dell laptop Latitude 2120.

\section{Statistical analysis}

Data was expressed as mean \pm SD. One-way ANOVA was used to test for the statistical difference between more than two groups. Dunnett's post hoc test was used to test were the significance lies. SPSS version 21 was used. $\mathrm{p}<0.05$ was considered as statistically significant

\section{Results}

Table 2 shows the presences of phytochemical substances in Salacia reticulate. It also shows that Saponins are more abundant in concentration than other chemicals.

\begin{tabular}{|l|c|c|c|}
\hline $\begin{array}{l}\text { Serial } \\
\text { number }\end{array}$ & Constituents & Tests & Inference \\
\hline 1 & Carbohydrate & Molisch test & + \\
\hline 2 & Anthraquinones & Bontragers test & + \\
\hline 3 & $\begin{array}{c}\text { Cardiac } \\
\text { glycosides }\end{array}$ & Kelle killani test & + \\
\hline 4 & Saponins & Frothing test & +++ \\
\hline 5 & $\begin{array}{c}\text { Steroids and } \\
\text { Triterpene }\end{array}$ & $\begin{array}{c}\text { Liberman Buchard } \\
\text { test }\end{array}$ & + \\
\hline 6 & Tannins & Fervic Chloride test & + \\
\hline 7 & Flavonoids & Shinodes test & + \\
\hline 8 & Alkaloids & Dragondorff Test & + \\
\hline \multicolumn{4}{|c|}{ I. } \\
\hline \multicolumn{4}{|c|}{ Key: + present; $-=$ absent; +++ abundance } \\
\hline
\end{tabular}

Table 2: Phytochemical result.

\section{Morphological result [body weight]}

Table 3, administration of aluminum chloride, or Salacia reticulata or Vitamin $\mathrm{E}$ affected the weight of the animal. There was a significant decrease in weight of animals in group B, C and $D$, while Group A and E weight increased. There was a significant difference at $\mathrm{p}<0.05$ between the Aluminum chloride and Salacia reticulata treated groups (C, and D) and the normal control group, which implies that aluminum chloride reduced the weight of the rats. It was observed that animals in Group C and D (Salacia reticulata treated groups), lost their weights after 14 days of treatment, while animals in Group E (vitamin E treated group) gained weight in course of treatment. (Table 4)

\begin{tabular}{|l|c|c|}
\hline Groups (n=5) & Initial weight & Final weight \\
\hline $\mathrm{A}$ & $73.60 \pm 2.50$ & $81.60 \pm 2.70$ \\
\hline $\mathrm{B}$ & $79.00 \pm 2.64$ & $77.20 \pm 1.92$ \\
\hline $\mathrm{C}$ & $74.80 \pm 3.56$ & $71.20 \pm 4.32^{*}$ \\
\hline $\mathrm{D}$ & $76.80 \pm 4.43$ & $72.80 \pm 4.76^{*}$ \\
\hline $\mathrm{E}$ & $78.40 \pm 2.70$ & $81.60 \pm 2.30$ \\
\hline $\begin{array}{l}\text { The values of the mean body of wistar rats in grams were ex- } \\
\text { pressed as mean } \pm \text { standard deviation (SD). }\end{array}$ = p<0.05 . Group A: \\
$\begin{array}{l}\text { Normal control; Group B: Alcl }{ }_{3} \text { control; Group C: Alcl }+250 \mathrm{mg} / \\
\text { kgbw salacia reticulata; Group D: Alcl }\end{array}$ + 300mg/kgbw salacia re- \\
ticulate; Group E: shows a level of significance.
\end{tabular}

Table 3: Mean initial and final body weight of adult male wistar rat.

\begin{tabular}{|c|c|}
\hline Groups (n=5) & Brain \\
\hline A & $1.48 \pm 0.04$ \\
\hline B & $1.44 \pm 0.08$ \\
\hline C & $1.48 \pm 0.04$ \\
\hline D & $1.50 \pm 0.00$ \\
\hline E & $1.50 \pm 0.14$ \\
\hline
\end{tabular}

Table 4: Mean brain weight of adult male wistar rat. 
Behavioural performance

Tables 5 and 6, showed a decrease in the meantime taken by the experimental animals to make alternations and to explore new arms in the y-maze test especially in Group B ( $\mathrm{Alcl}_{3}$ only) and also in group $\mathrm{C}\left(\mathrm{Alcl}_{3}+250 \mathrm{mg} / \mathrm{kgbw}\right.$ salacia reticulata $)$.

\begin{tabular}{|l|c|c|c|}
\hline Groups (n=5) & SABPT (\%) & SABPT (1 \%) & SABPT (2 \%) \\
\hline A & $69.00 \pm 4.84$ & $54.80 \pm 8.54$ & $56.20 \pm 8.37$ \\
\hline B & $61.00 \pm 4.71$ & $49.00 \pm 6.51^{*}$ & $45.80 \pm 6.97^{*}$ \\
\hline C & $66.80 \pm 7.70$ & $54.00 \pm 11.40$ & $57.80 \pm 5.40$ \\
\hline D & $70.00 \pm 3.53$ & $52.00 \pm 6.12$ & $54.00 \pm 6.51$ \\
\hline E & $68.80 \pm 5.61$ & $50.00 \pm 8.36^{*}$ & $53.36 \pm 8.36^{*}$ \\
\hline
\end{tabular}

The values of the mean body of wistar rats in grams were expressed as mean \pm standard deviation (SD). ${ }^{*}=p<0.05$. Group A: Normal control; Group B: $\mathrm{Alcl}_{3}$ control; Group C: $\mathrm{Alcl}_{3}+250 \mathrm{mg} /$ kgbw salacia reticulata; Group D: $\mathrm{Alcl}_{3}+300 \mathrm{mg} / \mathrm{kgbw}$ salacia reticulate; Group E: shows a level of significance.

SABPT $\%=$ Spontaneous alternation behaviour test

Table 5: Spontaneous alternation test.

\begin{tabular}{|c|c|c|c|}
\hline Groups (n=5) & NAEPT & NAET1 & NAET2 \\
\hline A & $8.40 \pm 2.88$ & $10.40 \pm 3.20$ & $12.60 \pm 2.60$ \\
\hline B & $11.00 \pm 5.78$ & $8.80 \pm 2.48$ & $5.80 \pm 1.30^{*}$ \\
\hline C & $7.40 \pm 1.51$ & $6.20 \pm 3.96$ & $6.40 \pm 3.75^{*}$ \\
\hline D & $9.00 \pm 3.46$ & $5.60 \pm 0.54$ & $7.40 \pm 2.50$ \\
\hline E & $11.20 \pm 2.38$ & $9.00 \pm 1.87$ & $9.40 \pm 2.28$ \\
\hline
\end{tabular}

The values of the mean body of wistar rats in grams were expressed as mean \pm standard deviation (SD). ${ }^{*}=p<0.05$. Group A; Normal control; Group B: $\mathrm{Alcl}_{3}$ control; Group C: $\mathrm{Alcl}_{3}+250 \mathrm{mg} /$ kgbw salacia reticulata; Group D: $\mathrm{Alcl}_{3}+300 \mathrm{mg} / \mathrm{kgbw}$ salacia reticulate; Group E: shows a level of significance.

NAEPT $=$ Number of arm entering test

Table 6: Number of arm entering.

\section{Biochemical Results}

AST, ALT, ALP levels were elevated significantly $(\mathrm{p}<0.05)$ in Alcl3 when compared with normal control (group A). salacia reticulata and vitamin E treated group expressed significantly ( $\mathrm{p}$ $<0.05$ ) reduced level of ALT and AST when compared to normal group. Salacia reticulata and Vitamin E stabilizes liver enzyme level in Group C, D and E, while Group B showed high level. A significant difference was observed between Group A and Group B. (Table 7)

Values expressed as mea enzyme \pm SD of the serum liver (U/L) of wistar rats during the experiment. Values with superscript in a column are significantly different $(\mathrm{p}<0.05)$. A; Normal Control; B Alcl3; Group C Alcl3+ 250mg/kgbw salacia extract; Group D Alcl3+ 300mg/kgbw salacia reticulata; Group E Alcl3+ Vitamin E. T-protein; Albumin; Globulin.(Table 8)

\begin{tabular}{|c|c|c|c|}
\hline Groups (n=5) & AST (U/L) & ALT (U/L) & ALP (U/L) \\
\hline A & $67.60 \pm 3.61$ & $45.80 \pm 5.31$ & $105.8 \pm 4.43$ \\
\hline B & $79.90 \pm 1.00$ & $62.80 \pm 2.86$ & $125.2 \pm 2.4$ \\
\hline C & $66.20 \pm 1.64$ & $46.00 \pm 5.14$ & $89.00 \pm 1.41$ \\
\hline D & $69.60 \pm 5.54$ & $48.80 \pm 1.78$ & $93.60 \pm 2.50^{*}$ \\
\hline e & $69.20 \pm 1.64$ & $46.80 \pm 0.83$ & $88.60 \pm 1.34^{*}$ \\
\hline
\end{tabular}

The values of the mean body of wistar rats in grams were expressed as mean \pm standard deviation (SD). ${ }^{*}=p<0.05$. Group A: Normal control; Group B: $\mathrm{Alcl}_{3}$ control; Group C: $\mathrm{Alcl}_{3}+$ $250 \mathrm{mg} / \mathrm{kgbw}$ salacia reticulata; Group D: $\mathrm{Alcl}_{3}+300 \mathrm{mg} / \mathrm{kgbw}$ salacia reticulate; Group E: shows a level of significance. AST: Aspartate aminotransferase; AlT: Alanine aminotransferase; ALP: Alkaline phosphatase.

Table 7: Mean of liver serum enzyme test.

\begin{tabular}{|l|c|c|c|}
\hline $\begin{array}{l}\text { Groups } \\
\text { (n=5) }\end{array}$ & T-protein (g/d) & Albumin (g/l) & Globulin (g/l) \\
\hline A & $5.66 \pm 0.05$ & $3.82 \pm 0.83$ & $105.8 \pm 4.43$ \\
\hline B & $5.22 \pm 34.2$ & $3.94 \pm 0.19$ & $125.2 \pm 2.4^{*}$ \\
\hline C & $5.70 \pm 0.10$ & $4.20 \pm 0.10$ & $89.00 \pm 1.41$ \\
\hline D & $5.76 \pm 0.05$ & $0.39 \pm 0.54$ & $93.60 \pm 2.50$ \\
\hline E & $5.64 \pm 0.54$ & $0.42 \pm 0.10$ & $88.60 \pm 1.34$ \\
\hline
\end{tabular}

Table 8: Mean value of T-protein, Albumin and Globulin.

\section{Histological Results}

Heamatoxylin and Eosin Stain [9].

The normal group (Plate 1) shows normal cellular profile and neutrophil there was no observable neuronal damage. The Alcl3 control group B (Plate 2) shows full neuronal damage to the brain cells and neutrophil (14 days). Group C (Plate 3) shows partial restoration of cellular and neutrophil profile with Salacia reticulate treated for (14days). Group D (Plate 4) shows full recovery of cellular and neutrophil profile (14 days). Group E (Plate 5) shows partial recovery of neural cells and cerebral cortex profile.

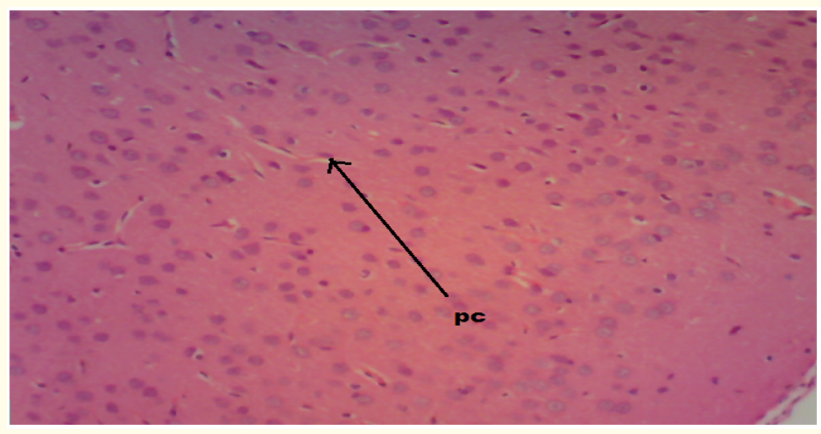

Plate 1: Photomicrograph of the cerebral cortex Group A, normal Cellular profile(PC) (H\&E) x100. 


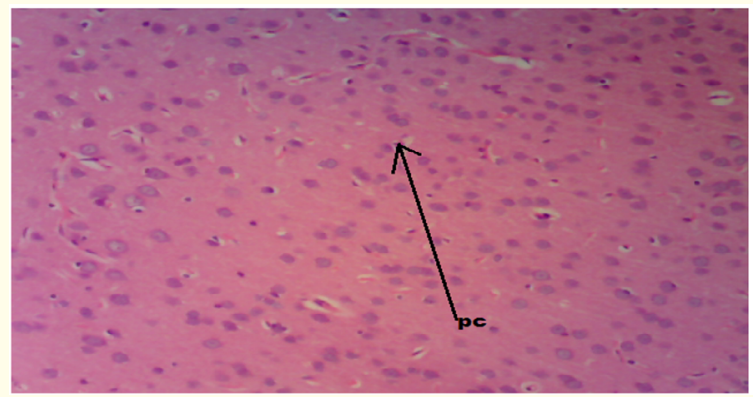

Plate 2: Photomicrograph of the cerebral cortex of Group B, degeneration of cellular and neutrophil profile (PC)

(H\&E)x100.

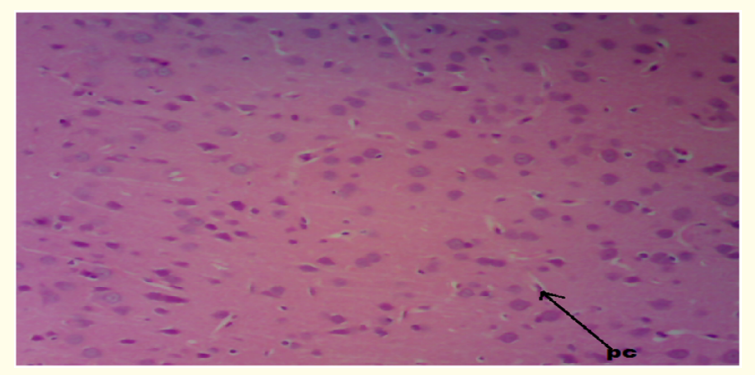

Plate 3: Photomicrograph of the cerebral cortex Group C, partial recovering of cellular and neutrophil profile (PC) (H\&E)x100



Plate 4: Photomicrograph of the cerebral cortex group D, shows full recovery of cellular and neutrophil (PC) (H\&E)x100

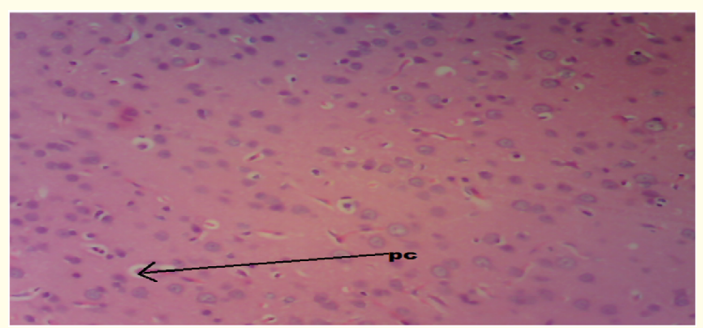

Plate 5: Shows partial recovery of cellular and neutrophil (H\&E) x100 on the cerebral cortex
Silver stain [10].

The normal group A (Plate 6) shows normal pyramidal cells and neutrophil there was no observable neuronal damage. The $\mathrm{Alcl}_{3}$ control group B (Plate 7) shows full degeneration of pyramidal cells and neutrophil (14 days). Group C (Plate 8) shows partial restoration of pyramidal cells and neutrophil profile with salacia reticulata treated for (14 days). Group D (Plate 9) shows full recovery of pyramidal cell and neutrophil profile. Group E shows partial recovery using vitamin for administration for 14 days. (Plate 10)

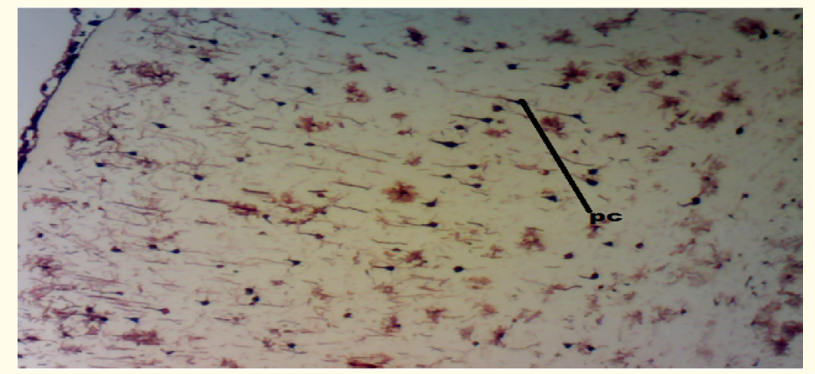

Plate 6: Photomicrograph of the cerebral cortex Group $\mathrm{A}$ (control), normal pyramidal cell (PC)(H\&E)x100.

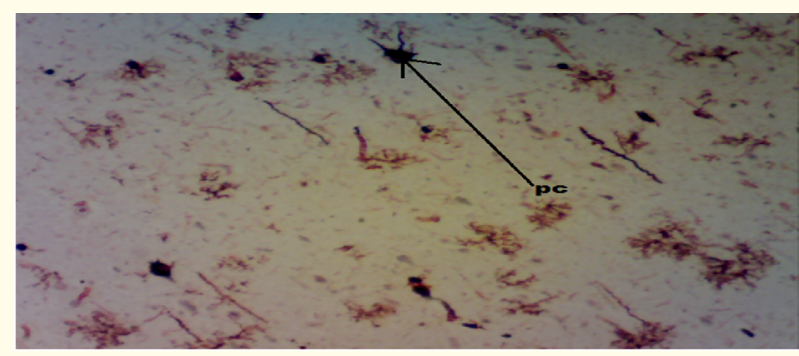

Plate 7: Photomicrograph of the cerebral cortex Group B, degeneration of pyramidal cell(H\&E)x100.



Plate 8: Photomicrograph of the cerebral cortex Group C, partial recovery of pyramidal cell (PC)(H\&E)x100. 


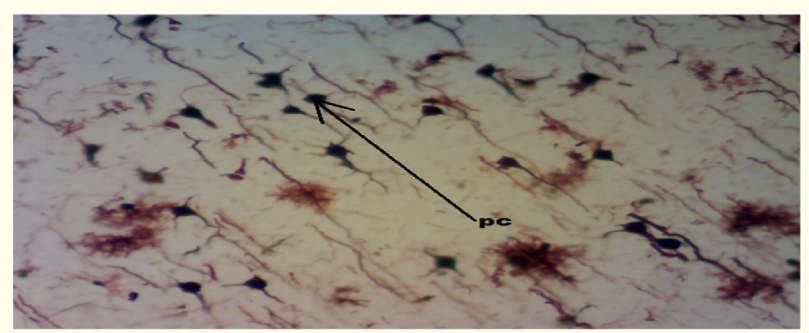

Plate 9: Photomicrograph of the cerebral cortex Group D, showing recovery of the Pyramidal cells (PC), (H\&E)x100.

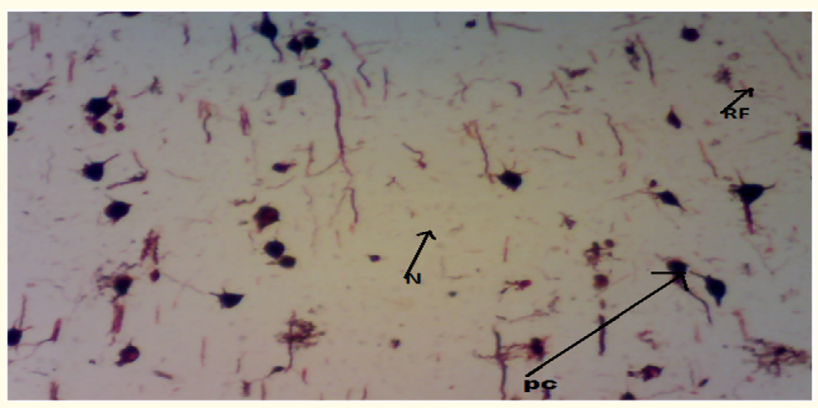

Plate 10: Photomicrograph of the cerebral cortex Group E, partial regeneration of pyramidal cells (PC), neuropin(N), radial fibers (RF), (H\&E) x100.

\section{Discussion}

Morphological result

Table 3, Administration of aluminium chloride and treatment with Salacia reticulata and Vitamin E affected the body weight of experimental animals. There was a significant decrease in weight of animals in group B, C and D, while in Group A and E weight increased. The findings of [11] were in agreement with this study.

\section{Behavioural performance}

The study showed a decrease in the meantime taken by the experimental animals to make alternations and to explore new arms in the y-maze test as shown in Tables 5 and 6 . The decrease in time taken for animals in group B to explore arms when compared with animals in group A could be as a result of anxiety which is associated with aluminium treatment [12]. Salacia reticulata administration was able to increase the time even better than vitamin E, implying that Salacia reticulate is able to alleviate anxiety.

\section{Histological Results}

The normal group (plate1) shows normal cellular profile and neutrophil there was no observable neuronal damage. The
$\mathrm{Alcl}_{3}$ control group B (plate2) shows full neuronal damage to the brain cells and neutrophil (14days). This supports a hypothetical statement by [13]. that Aluminium exposure has neurodegenerating effect resulting in learning deficits. Group C (plate 3) shows partial restoration of cellular and neutrophil profile with Salacia reticulate treated for (14days). Group D (Plate 4) shows full recovery of cellular and neutrophil profile (14days). Group E (plate 5) shows partial recovery of neural cells and cerebral cortex profile. Salacia reticulata treated groups (Plate 3 and 4), showed restoration of cellular and neutrophil profile when compared with Plate 2 ( $\mathrm{Alcl}_{3}$ treated groups) where the cells are damaged; this confirms that Salacia reticulata has neuroprotective effects on brain cells. Plate 5 (vitamin E treated group), showed partial recovery when compared with Plate 4 (Salacia reticulata treated groups), which showed full recovery of cellular profile and preservation of neuronal cells, implying that more antioxidants properties found in Salacia reticulata than in vitamin E. The result from this study is in agreement with the findings of [14].

The normal group A (plate 6) shows normal pyramidal cells and neutrophil there was no observable neuronal damage. The $\mathrm{Alcl}_{3}$ control group B (plate 7) shows full degeneration of pyramidal cells and neutrophil (14 days). Group C (plate 8) shows partial restoration of pyramidal cells and neutrophil profile with Salacia reticulata treated for (14 days). Group D (Plate 9) shows full recovery of pyramidal cell and neutrophil profile. Group E shows partial recovery using vitamin E for administration for 14days. The results collaborate with [15]. They reported that ethanolic root extract of Salacia reticulate improves memory and cognition in Mercury Chloride induced cellular damage of the hippocampus adult male mice.

\section{Biochemical results}

In group $\mathrm{B}\left(\mathrm{Alcl}_{3}\right), \mathrm{AST}, \mathrm{ALT}, \mathrm{ALP}$ increased rapidly, which implies that aluminium chloride treatment has damaged the liver. This result is in agreement with [11], they reported congested central vein and distorted sinusoids in the liver of wistar rats due to aluminium chloride exposure. Salacia reticulata and vitamin E treated group expressed significantly $(\mathrm{p}<0.05)$ reduced level of ALT and AST when compared to normal group, which implies that Salacia reticulata and Vitamin E are capable of stabilizing liver enzyme. Total protein, Albumin and globulin shows that the Aluminium chloride treatment affected the biochemical parameters negatively while Salacia reticulata treatment attenuates these parameters resulting in the positive improvement in the animal behaviour decreasing anxiety.

\section{Conclusion}

From the results obtained, Aqueous Crude Root Extract of Salacia reticulata at the above recommended dosage has 
Neuroprotective properties and is capable of alleviating anxiety. Salacia reticula also stabilizes liver enzymes and attenuates total protein, globulin and albumin levels in aluminium chloride $\left(\mathrm{AlCl}_{3}\right)$ induced cellular damage the cerebral cortex in Wistar rats.

\section{Bibliography}

1. Pavlov PI "Conditioned reflexes: An investigation of the physiological activities of the cerebral cortex". Annals of Neurosciences 17.3 (2010): 136-141.

2. Brodal S. "The Central Nervous Sys-tem: Structure and Function". Oxford University Press, Oxford (2012).

3. Cauller L. "Layer I of primary sensory neocortex: where topdown". Convergence Chemical Toxicology 41 (1995): 867-874.

4. World Health Organization (WHO). "Guidelines for DrinkingWater Quality: Recommendations". World Health Organization: Geneva, Switzerland (2011).

5. Sher Ali Khan., et al. "Levels of selected heavy metals in drinking water in Peshawar City". International Journal of Science and Nature 2.3 (2011): 648-652.

6. Gebrekidan Mebrahtu and Samuel Zerabruk. "Concentration of heavy metals in drinking water from urban areas of Tigray Region Northern". Memona Ethiopian Journal of Science 3.1 (2011): 105-121.

7. Trease GE and Evans WC. "Methods of phytochemical screening in plants". Pharmacognosy (15 ${ }^{\text {th }}$ Ed), WB Saunders, London (2002).

8. Stanford Medicine. "Behavioural and functional neuroscience laboratory manual of behavioural studies". Y maze study test protocol (2017).

9. Bancroft DJ and Gamble M. "Haematoxylin and Eosin staining method (Mayers 1903)". Theory and Practice of Histological Techniques (6 ${ }^{\text {th }}$ edn) (2008): 123-127.

10. Bancroft DJ and Gamble M. "Bielschowsky's silver stain for neurofibrils, dendrites, and axons in paraffin and frozen sections, modified (Chan \& Lowe 2002)". Theory and practice of Histological Techniques ( $6^{\text {th }} \mathrm{edn}$ ) (2008): 369-371.

11. Buraimoh AA and Ojo SA. "Effects of Aluminium chloride exposure on the body weight of Wistar rats". Annals of Biological Sciences 2.2 (2014): 66-73.

12. Buraimoh AA., et al. "Effects of Oral Administration of Aluminium Chloride on the Histology of the Hippocampus of Wistar Rats". Current Research Journal of Biological Sciences 3.5 (2011): 509-515.
13. Buraimoh AA., et al. "Behavioural Endpoints of Adult Wistar Rats, Following Aluminium Chloride Exposure". British Journal of Pharmacology and Toxicology 2.5 (2011a): 273-276. ISSN: 2044- 2467.

14. Yokel RA. "Repeated systemic aluminium exposure effects on classical conditioning of the rabbit". Neurotoxicology and Teratology 5 (1983): 41-46.

15. Godam Elvis Tams., et al. "Antidegenerative and Neurobehavioral Effects of Ethanolic Root Extract of Salacia reticulata on Mercury Chloride Induced Cellular Damage in the Hippocampus of Adult Male Mice". Journal of Cytology and Histology 9.3 (2018): 508.

Volume 3 Issue 4 April 2019

(C) All rights are reserved by Elvis Tams Godam., et al. 